\title{
The twisted products of spheres that have the fixed point property
}

\author{
by \\ Haibao Duan and Boju Jiang (Beijing)
}

\begin{abstract}
By a twisted product of $S^{n}$ we mean a closed, 1-connected $2 n$-manifold $M$ whose integral cohomology ring is isomorphic to that of $S^{n} \times S^{n}, n \geq 3$. We list all such spaces that have the fixed point property.
\end{abstract}

1. Introduction. An almost smooth manifold is a pair $\left(M, D_{M}\right)$ in which

(1) $M$ is a closed, 1-connected topological manifold;

(2) $D_{M} \subset M$ is an embedded $\operatorname{disc}, \operatorname{dim} D_{M}=\operatorname{dim} M$; and

(3) $M \backslash \operatorname{int} D_{M}$ is furnished with a fixed smooth structure.

A homeomorphism between two almost smooth manifolds $\left(M, D_{M}\right)$ and $\left(N, D_{N}\right)$ is a homeomorphism $F: M \rightarrow N$ that restricts to a diffeomorphism

$$
M \backslash \operatorname{int} D_{M} \rightarrow N \backslash \operatorname{int} D_{N} .
$$

It is clear that such homeomorphisms yield an equivalence relation among all almost smooth manifolds. Denote by $W$ the set of equivalence classes of this relation.

The category $W$ introduced above is of classical interest. C. T. C. Wall classified all $(n-1)$-connected $2 n$ - and $(2 n+1)$-manifolds, $n \geq 3$, exactly in this category $\left[\mathrm{W}_{1}\right],\left[\mathrm{W}_{2}\right]$. In general, it may be considered as a category between the smooth and PL categories.

It has been forty years since a complete classification for $(n-1)$-connected $2 n$-dimensional almost smooth manifolds was achieved by C. T. C. Wall $\left[\mathrm{W}_{1}\right]$. It seems, however, that the corresponding investigation into the geometry of maps between such manifolds has not yet received as much attention

2000 Mathematics Subject Classification: Primary 55M20; Secondary 57N15.

Key words and phrases: fixed point property, Lefschetz number, J-homomorphism.

Research of B. J. Jiang supported by MoSTC and MoEC grants. 
as it deserves. In this paper, without attempting a thorough study of this broad subject, we just present an evidence indicating an interesting aspect of this topic in the context of fixed point theory.

A topological space $X$ is said to have the fixed point property if the equation

$$
f(x)=x, \quad x \in X,
$$

has a solution for every self-map $f$ of $X$. The classical Brouwer fixed point theorem asserts that the $n$-dimensional disc $D^{n}=\left\{x \in \mathbb{R}^{n}|| x \mid \leq 1\right\}$ has the fixed point property. During the past century it has served as one of the main technical tools in establishing existence results for highly nonlinear problems $[\mathrm{Fo}]$. On the other hand, except for even dimensional projective spaces and certain complex Grassmannians, few examples of closed manifolds are known to have this striking but useful property $[\mathrm{F}],[\mathrm{H}]$.

By a twisted product of $S^{n}, n \geq 3$, we mean a closed, 1-connected, almost smooth $2 n$-manifold $M$ whose integral cohomology ring is isomorphic to that of $S^{n} \times S^{n}$. The importance and generality of such spaces can be seen from the following facts due to Wall $\left[\mathrm{W}_{1}\right]$. Let $S(n)$ be the set of all homeomorphism types of twisted products of $S^{n}$. Then

(i) if $n$ is odd, connected sums of elements in $S(n)$ yield all almost smooth $(n-1)$-connected $2 n$-manifolds;

(ii) if $n$ is even and if $n \neq 4,8$, the Grothendieck group of $n$-spaces is generated by elements in $S(n)$ together with the single $n$-space whose intersection form is given by $E_{8}$ (cf. Theorem 2 in $\left[\mathrm{W}_{1}\right]$ ).

The standard product $S^{n} \times S^{n}$ clearly fails the fixed point property. However, this is no longer so for twisted products of $S^{n}$.

In order to describe our results, we need some notation to describe the homotopy type of manifolds in $S(4 k)$. First recall that the Bernoulli numbers $B_{k}$ are the rationals defined by

$$
\frac{x}{e^{x}-1}=1-\frac{x}{2}+\sum_{k \geq 1}(-1)^{k-1} B_{k} \frac{x^{2 k}}{(2 k) !} .
$$

Let $d_{k}$ be the denominator of $B_{k} / 4 k$ (expressed in lowest terms). Put $\sigma_{k}=$ $d_{k} / 2$ if $k=1,2$ and let $\sigma_{k}=d_{k}$ if $k \geq 3$. The first 10 values of $\sigma_{k}$ are

\begin{tabular}{cccccccccc}
\hline$\sigma_{1}$ & $\sigma_{2}$ & $\sigma_{3}$ & $\sigma_{4}$ & $\sigma_{5}$ & $\sigma_{6}$ & $\sigma_{7}$ & $\sigma_{8}$ & $\sigma_{9}$ & $\sigma_{10}$ \\
12 & 120 & 504 & 480 & 264 & 65520 & 24 & 16320 & 28728 & 13200
\end{tabular}

It is shown in Section 4 that the set $S(4 k)$ is indexed by pairs of integers as

$$
S(4 k)=\{M(a, b) \mid a, b \in \mathbb{Z}\},
$$


where, with respect to a certain basis $x, y$ for $H^{4 k}(M(a, b))=\mathbb{Z} \oplus \mathbb{Z}$, the parameter $(a, b)$ is related to the Pontryagin class $p_{k}$ for the stable tangent bundle of $M(a, b)$ by

$$
p_{k}=2^{\varepsilon(k)}(2 k-1) !(a x+b y), \quad \varepsilon(k)= \begin{cases}0 & \text { if } k \text { is even }, \\ 1 & \text { if } k \text { is odd }\end{cases}
$$

A homotopy classification of elements of $S(4 k)$ is given in

Theorem 2. $M\left(a_{1}, a_{2}\right)$ is homotopy equivalent to $M\left(b_{1}, b_{2}\right)$ if and only if one of the following eight congruence systems is satisfied:

$$
\left\{\begin{array} { l } 
{ a _ { 1 } \pm b _ { 1 } \equiv 0 ( \operatorname { m o d } \sigma _ { k } ) , } \\
{ a _ { 2 } \pm b _ { 2 } \equiv 0 ( \operatorname { m o d } \sigma _ { k } ) , }
\end{array} \quad \left\{\begin{array}{l}
a_{1} \pm b_{2} \equiv 0\left(\bmod \sigma_{k}\right) \\
a_{2} \pm b_{1} \equiv 0\left(\bmod \sigma_{k}\right)
\end{array}\right.\right.
$$

Consequently, the subset $T(k)=\left\{M\left(a_{1}, a_{2}\right) \mid 0 \leq a_{1} \leq a_{2} \leq \sigma_{k} / 2\right\}$ of $S(4 k)$ consists of all distinct homotopy types of twisted products of $S^{4 k}$.

Since the fixed point property is invariant with respect to homotopy equivalence of closed manifolds, a combination of Theorem 2 with the next result classifies, with respect to homeomorphism type, all twisted products of $S^{n}$ that have the fixed point property.

For $a \in \mathbb{Z}$ let $o(a) \in \mathbb{Z}$ be the order of $a$ in the cyclic group $\mathbb{Z}_{\sigma_{k}}$.

Theorem 3. If $n=4 k$, then $M=M\left(a_{1}, a_{2}\right) \in T(k)$ has the fixed point property if and only if $a_{1} a_{2} \neq 0$ and

$$
\begin{array}{r}
\operatorname{gcd}\left\{o\left(a_{1}\right), o\left(a_{2}\right)\right\} \neq 1, \\
\operatorname{gcd}\left\{2 o\left(a_{1}\right), o\left(a_{2}\right)\right\} \neq 2, \\
\operatorname{gcd}\left\{o\left(a_{1}\right), 2 o\left(a_{2}\right)\right\} \neq 2 .
\end{array}
$$

If $n \neq 4 k$, then every $M \in S(n)$ fails the fixed point property.

Let $J(k)$ be the subset of $T(k)$ consisting of all the homotopy types that have the fixed point property, and let $c_{k}$ be the cardinality of $J(k)$. Computation based on Theorem 3 shows that most of the elements in $T(k)$ have the fixed point property.

Table 1. $J(1)$

\begin{tabular}{lllll}
\hline$(1,1)$ & & & & \\
$(1,2)$ & $(2,2)$ & & & \\
$(1,3)$ & & $(3,3)$ & & \\
$(1,4)$ & $(2,4)$ & & $(4,4)$ & \\
$(1,5)$ & $(2,5)$ & $(3,5)$ & $(4,5)$ & $(5,5)$ \\
\hline
\end{tabular}


Table 2. $J(7)$

\begin{tabular}{lllllllllll}
\hline$(1,1)$ & & & & & & & & & & \\
$(1,2)$ & $(2,2)$ & & & & & & & & \\
$(1,3)$ & $(2,3)$ & $(3,3)$ & & & & & & & \\
$(1,4)$ & $(2,4)$ & & $(4,4)$ & & & & & & \\
$(1,5)$ & $(2,5)$ & $(3,5)$ & $(4,5)$ & $(5,5)$ & & & & & \\
$(1,6)$ & $(2,6)$ & $(3,6)$ & & $(5,6)$ & $(6,6)$ & & & & & \\
$(1,7)$ & $(2,7)$ & $(3,7)$ & $(4,7)$ & $(5,7)$ & $(6,7)$ & $(7,7)$ & & & & \\
$(1,8)$ & $(2,8)$ & & $(4,8)$ & $(5,8)$ & & $(7,8)$ & $(8,8)$ & & & \\
$(1,9)$ & $(2,9)$ & $(3,9)$ & & $(5,9)$ & $(6,9)$ & $(7,9)$ & & $(9,9)$ & & \\
$(1,10)$ & $(2,10)$ & $(3,10)$ & $(4,10)$ & $(5,10)$ & $(6,10)$ & $(7,10)$ & $(8,10)$ & $(9,10)$ & $(10,10)$ & \\
$(1,11)$ & $(2,11)$ & $(3,11)$ & $(4,11)$ & $(5,11)$ & $(6,11)$ & $(7,11)$ & $(8,11)$ & $(9,11)$ & $(10,11)$ & $(11,11)$ \\
\hline
\end{tabular}

Table 3. $c_{k}, k \leq 10$

\begin{tabular}{cccccc}
\hline$k$ & 1 & 2 & 3 & 4 & 5 \\
$c_{k}$ & 13 & 1672 & 31104 & 28222 & 8410 \\
\hline$k$ & 6 & 7 & 8 & 9 & 10 \\
$c_{k}$ & 469532700 & 60 & 33250102 & 103080204 & 21744712 \\
\hline
\end{tabular}

For $M \in S(n)$ denote by $H_{M}$ the cohomology $H^{n}(M ; \mathbb{Z})$ in the middle dimension.

The paper is organized as follows. Section 2 recalls from $\left[\mathrm{W}_{1}\right]$ the constructions of elements in $S(n)$ both in terms of handle decomposition and cell decomposition. In Theorem 1 (Section 3) we answer the question which homomorphism $H_{N} \rightarrow H_{M}$ can be induced by a continuous map $f: M \rightarrow N$ with $M, N \in S(n)$. Combining Theorem 1 with the results of Adams [A] and Quillen [Q] of late 60's, we obtain in Section 4 a homotopy type classification for elements in $S(n)$, for $n$ even, which was incomplete in [ $\left.\mathrm{W}_{1}\right]$ due to lack of information on J-homomorphisms. The proof of Theorem 3 is given in Section 5; finally, Section 6 discusses some numerical phenomena arising from the previous computation.

2. Constructions. Let $J: \pi_{n-1}(S O(n)) \rightarrow \pi_{2 n-1}\left(S^{n}\right)$ be the J-homomorphism [Wh], and let $H: \pi_{2 n-1}\left(S^{n}\right) \rightarrow \mathbb{Z}$ be the Hopf invariant. We recall from $\left[\mathrm{W}_{1}\right.$ ] that elements in $S(n)$ are parameterized by pairs of elements in the group

$$
G_{n}=\operatorname{Ker}\left\{H \circ J: \pi_{n-1}(S O(n)) \rightarrow \mathbb{Z}\right\} .
$$

Let $D^{2 n}$ be the standard $2 n$-disc. Fix two smooth embeddings

$$
h_{i}: S^{n-1} \times D^{n} \rightarrow S^{2 n-1}=\partial D^{2 n} \subset D^{2 n}, \quad i=1,2,
$$

with disjoint images so that the linking number of the restrictions $h_{1} \mid S^{n-1} \times 0$ and $h_{2} \mid S^{n-1} \times 0$ in $S^{2 n-1}$ is 1 . For two $\alpha_{i} \in G_{n}, i=1,2$, let $N\left(\alpha_{1}, \alpha_{2}\right)$ be 
the handle body

$$
D^{2 n} \bigcup_{\alpha_{1}^{\prime} \sqcup \alpha_{2}^{\prime}}\left(D^{n} \times D^{n} \sqcup D^{n} \times D^{n}\right)
$$

with the attaching maps

$$
D^{n} \times D^{n} \supset \partial\left(D^{n} \times D^{n}\right) \supset S^{n-1} \times D^{n} \stackrel{\alpha_{i}^{\prime}}{\longrightarrow} S^{2 n-1}=\partial D^{2 n} \subset D^{2 n}
$$

defined by $\alpha_{i}^{\prime}(x, y)=h_{i}\left(x, \alpha_{i}(x) y\right), i=1,2$. Then $N\left(\alpha_{1}, \alpha_{2}\right)$ is a smooth manifold whose boundary is topologically a $(2 n-1)$-sphere (cf. Corollary to Lemma 3 in $\left.\left[\mathrm{W}_{1}\right]\right)$, so a $2 n$-dimensional disc $D^{2 n}$ can be added to yield a closed almost smooth $2 n$-manifold $M\left(\alpha_{1}, \alpha_{2}\right)=\left(N\left(\alpha_{1}, \alpha_{2}\right) \cup D^{2 n}, D^{2 n}\right)$. Since $M\left(\alpha_{1}, \alpha_{2}\right)$ is simply connected (because $\left.n \geq 3\right)$ and its intersection form is seen to be

$$
\left(\begin{array}{cc}
0 & (-1)^{n} \\
1 & 0
\end{array}\right)
$$

it follows that $M\left(\alpha_{1}, \alpha_{2}\right) \in S(n)$. Conversely, all elements in $S(n)$ are obtained in this way.

The space $M\left(\alpha_{1}, \alpha_{2}\right)$ admits a cell decomposition

$$
M\left(\alpha_{1}, \alpha_{2}\right)=\bigvee_{i=1,2} S_{i}^{n} \cup_{\alpha} D^{2 n},
$$

with the attaching map $\alpha \in \pi_{2 n-1}\left(\bigvee_{i=1,2} S_{i}^{n}\right)$ related to $\alpha_{1}, \alpha_{2} \in G_{n} \subseteq$ $\pi_{n-1}(S O(n))$ as follows. Let $\iota_{i}: S^{n} \rightarrow \bigvee_{i=1,2} S_{i}^{n} \subset M\left(\alpha_{1}, \alpha_{2}\right)$ be the inclusion onto the $i$ th copy of the bouquet $\bigvee_{i=1,2} S_{i}^{n}, i=1,2$. By a result of Hilton, there is a canonical splitting

$$
\pi_{2 n-1}\left(\bigvee_{i=1,2} S_{i}^{n}\right)=\bigoplus_{i=1,2} \pi_{2 n-1}\left(S_{i}^{n}\right) \oplus \pi_{2 n-1}\left(S^{2 n-1}\right) .
$$

Lemma 1. With respect to the splitting (2.1), $\alpha=\iota_{1} \circ J\left(\alpha_{1}\right)+\iota_{2} \circ J\left(\alpha_{2}\right)+$ $\left[\iota_{1}, \iota_{2}\right]$, where [,] stands for the Whitehead product $\left[\mathrm{W}_{1}\right]$, [Wh].

REMARK 1. It follows from Lemma 1 that $\pi_{r}\left(M\left(\alpha_{1}, \alpha_{2}\right)\right) \cong \pi_{r}\left(S^{n} \times S^{n}\right)$, $r \geq 0$.

3. Realization of a cohomology homomorphism by a map. For two $M, N \in S(n)$, sending a continuous map $f: M \rightarrow N$ to the induced cohomology homomorphism yields a representation

$$
r:[M, N] \rightarrow \operatorname{Hom}\left(H_{N}, H_{M}\right),
$$

where $[M, N]$ is the set of all homotopy classes of maps $M \rightarrow N$. This section is devoted to a description of $\operatorname{Im}(r)$, the image of $r$ in $\operatorname{Hom}\left(H_{N}, H_{M}\right)$.

Assume, by the discussion in the previous section, that

$$
M=M\left(\alpha_{1}, \alpha_{2}\right)=\bigvee_{i=1,2} S_{i}^{n} \cup_{\alpha} D^{2 n}, \quad N=M\left(\beta_{1}, \beta_{2}\right)=\bigvee_{i=1,2} S_{i}^{n} \cup_{\beta} D^{2 n}
$$


with $\iota_{i}: S^{n} \rightarrow \bigvee_{i=1,2} S_{i}^{n} \subset M$ (resp. $\left.\iota_{i}^{\prime}: S^{n} \rightarrow \bigvee_{i=1,2} S_{i}^{n} \subset N\right)$ being the inclusion onto the $i$ th component of the bouquet $\bigvee_{i=1,2} S_{i}^{n}, i=1,2$. Let $e_{i} \in H_{M}$ (resp. $e_{i}^{\prime} \in H_{N}$ ) be the image of $\iota_{i *}\left[S^{n}\right] \in H_{n}(M ; \mathbb{Z})$ (resp. $\iota_{i *}^{\prime}\left[S^{n}\right] \in H_{n}(N ; \mathbb{Z})$ ) under Poincaré duality. Then $H_{M}=\operatorname{span}\left\{e_{1}, e_{2}\right\}$ (resp. $\left.H_{N}=\operatorname{span}\left\{e_{1}^{\prime}, e_{2}^{\prime}\right\}\right)$. In view of this we may equally well regard $r$ as a representation into the set $M(2)$ of all $2 \times 2$ integer matrices,

$$
r:[M, N] \rightarrow M(2),
$$

by $r(f)=\left(a_{i j}\right)_{2 \times 2}$, where $f^{*}\left(e_{i}^{\prime}\right)=a_{i 1} e_{1}+a_{i 2} e_{2}, i=1,2$.

THEOREM 1. $A=\left(a_{i j}\right)_{2 \times 2} \in \operatorname{Im}(r)$ if and only if the following equations hold in $\pi_{2 n-1}\left(S^{n}\right)$ :

$$
k J\left(\beta_{i}\right)=a_{i 1} J\left(\alpha_{1}\right)+a_{i 2} J\left(\alpha_{2}\right)+a_{i 1} a_{i 2}\left[\kappa_{n}, \kappa_{n}\right], \quad i=1,2,
$$

where $k=a_{11} a_{22}+(-1)^{n} a_{12} a_{21}$, and where $\kappa_{n} \in \pi_{n}\left(S^{n}\right)$ is the class of the identity.

If $n$ is even, applying the Hopf invariant $H$ to (3.1) gives

$$
a_{11} a_{12}=a_{21} a_{22}=0
$$

(since $\alpha_{i}, \beta_{i} \in G_{n}$ and $H\left(\left[\kappa_{n}, \kappa_{n}\right]\right)=2$ ). Theorem 1 implies

Corollary 1. Let $n$ be even. Then $A=\left(a_{i j}\right)_{2 \times 2} \in \operatorname{Im}(r)$ if and only if one of the following constraints is satisfied:
(i) $A=\left(\begin{array}{ll}a & 0 \\ 0 & b\end{array}\right)$ with $\left\{\begin{array}{l}a b J\left(\beta_{1}\right)=a J\left(\alpha_{1}\right) \\ a b J\left(\beta_{2}\right)=b J\left(\alpha_{2}\right)\end{array}\right.$ in $\pi_{2 n-1}\left(S^{n}\right)$;
(ii) $A=\left(\begin{array}{ll}0 & b \\ a & 0\end{array}\right)$ with $\left\{\begin{array}{l}a b J\left(\beta_{1}\right)=b J\left(\alpha_{2}\right) \\ a b J\left(\beta_{2}\right)=a J\left(\alpha_{1}\right)\end{array}\right.$ in $\pi_{2 n-1}\left(S^{n}\right)$;
(iii) $A=\left(\begin{array}{ll}a & 0 \\ b & 0\end{array}\right)$ with $\left\{\begin{array}{l}a J\left(\alpha_{1}\right)=0 \\ b J\left(\alpha_{1}\right)=0\end{array}\right.$ in $\pi_{2 n-1}\left(S^{n}\right)$;
(iv) $A=\left(\begin{array}{ll}0 & a \\ 0 & b\end{array}\right)$ with $\left\{\begin{array}{l}a J\left(\alpha_{2}\right)=0 \\ b J\left(\alpha_{2}\right)=0\end{array}\right.$ in $\pi_{2 n-1}\left(S^{n}\right)$.

We complete this section by proving Theorem 1 . For a homomorphism $h: H_{N} \rightarrow H_{M}$ one constructs a map $g: \bigvee_{i=1,2} S_{i}^{n} \rightarrow \bigvee_{i=1,2} S_{i}^{n}$ so that the induced $g^{*}$ on cohomology fits in the commutative diagram

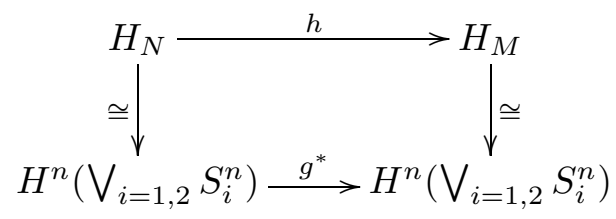

where the vertical isomorphisms are induced by the inclusions $\bigvee_{i=1,2} S_{i}^{n}$ $\subset N$ and $\bigvee_{i=1,2} S_{i}^{n} \subset M$. A standard discussion in homotopy theory yields 
Lemma 2. $g$ is extendible to a map $f: M \rightarrow N$ (of degree $k$ ) if and only if the induced homomorphism $g_{*}: \pi_{2 n-1}\left(\bigvee_{i=1,2} S_{i}^{n}\right) \rightarrow \pi_{2 n-1}\left(\bigvee_{i=1,2} S_{i}^{n}\right)$ satisfies

$$
g_{*}(\alpha)=k \beta \text {. }
$$

Assume that, with respect to the basis $\left\{e_{1}^{\prime}, e_{2}^{\prime}\right\}$ for $H_{N}$ and $\left\{e_{1}, e_{2}\right\}$ for $H_{M}, h: H_{N} \rightarrow H_{M}$ has the representation

$$
h\left(e_{i}^{\prime}\right)=\sum_{j=1,2} a_{i j} e_{j}, \quad a_{i j} \in \mathbb{Z} .
$$

Equivalently $g_{*}: \pi_{n}\left(\bigvee_{i=1,2} S_{i}^{n}\right) \rightarrow \pi_{n}\left(\bigvee_{i=1,2} S_{i}^{n}\right)$ is given by $g_{*}\left(\iota_{i}\right)=$ $\sum_{j=1,2} a_{j i} \iota_{j}^{\prime}, i=1,2$. With these notations we compute

$$
\begin{aligned}
g_{*}(\alpha)= & \sum_{i=1,2} g_{*}\left(\iota_{i}\right) \circ J\left(\alpha_{i}\right)+\left[g_{*}\left(\iota_{1}\right), g_{*}\left(\iota_{2}\right)\right] \\
= & \sum_{j=1,2} \iota_{j}^{\prime} \circ\left(a_{j 1} J\left(\alpha_{1}\right)+a_{j 2} J\left(\alpha_{2}\right)+a_{j 1} a_{j 2}\left[\kappa_{n}, \kappa_{n}\right]\right) \\
& +\left(a_{11} a_{22}+(-1)^{n} a_{12} a_{21}\right)\left[\iota_{1}^{\prime}, \iota_{2}^{\prime}\right],
\end{aligned}
$$

where we have made use of the $(-1)^{n}$-symmetry and bilinearity of the Whitehead product [,], the bilinearity of the composition operator $\circ$ (note that $\circ$ is linear with respect the first factor since $\alpha_{i} \in G_{n}$, cf. formula (1.16) in [Wh, p. 494]), as well as the obvious relation

$$
\left[\iota_{i}^{\prime}, \iota_{i}^{\prime}\right]=\iota_{i}^{\prime} \circ\left[\kappa_{n}, \kappa_{n}\right]
$$

(in $\pi_{2 n-1}\left(\bigvee_{i=1,2} S_{i}^{n}\right)$ ). Now comparing the coefficients of $\iota_{i}^{\prime}$ and $\left[\iota_{1}^{\prime}, \iota_{2}^{\prime}\right]$ on both sides of (3.2) yields

Lemma 3. $g$ is extendible to a map $f: M \rightarrow N$ (of degree $k$ ) if and only if the homomorphism $h$ defined by (3.3) satisfies

$$
k J\left(\beta_{i}\right)=a_{i 1} J\left(\alpha_{1}\right)+a_{i 2} J\left(\alpha_{2}\right)+a_{i 1} a_{i 2}\left[\kappa_{n}, \kappa_{n}\right], \quad i=1,2,
$$

in $\pi_{2 n-1}\left(S^{n}\right)$, where $k=a_{11} a_{22}+(-1)^{n} a_{12} a_{21}$.

This clearly finishes the proof of Theorem 1 .

4. Homotopy type classification in $S(n)$ (for $n$ even). Assume throughout this section that $n$ is even. We need information on the groups $G_{n}$, as well as the restriction of the J-homomorphism to $G_{n}$. In the statement and proof of the next result we use a section of the homotopy sequence

$$
\pi_{n}\left(S^{n}\right) \stackrel{\partial}{\rightarrow} \pi_{n-1}(S O(n)) \stackrel{i_{*}}{\rightarrow} \pi_{n-1}(S O(n+1)) \rightarrow 0
$$

of the fibration $S O(n+1) \rightarrow S^{n}$. The number $\sigma_{k}$ is as defined in Section 1 .

Lemma 4. The groups $G_{n}$ and the restriction of the J-homomorphism to $G_{n}$ can be classified into the following four cases. 
CASE 1. $n=4 k, k \leq 2$ : Let $\delta \in \pi_{n-1}(S O(n-1))=\mathbb{Z}$ be a generator and put $x=i_{*}(\delta)$. Then

(1-1) $G_{n}=\mathbb{Z}$ is generated by $x$;

(1-2) $J(x)$ generates a direct cyclic summand of $\pi_{2 n-1}\left(S^{n}\right)$ of order $\sigma_{k}$.

CASE 2. $n=4 k, k \geq 3:$ Let $y \in \pi_{n-1}(S O(n))$ be a class such that $i_{*}(y)$ generates $\pi_{n-1}(S O(n+1))=\mathbb{Z}$, and put $x=y-\frac{1}{2} H J(y) \partial \kappa_{n}$. Then

$(2-1) G_{n}=\mathbb{Z}$ is generated by $x$

$(2-2) J(x)$ generates a direct cyclic summand of $\pi_{2 n-1}\left(S^{n}\right)$ of order $\sigma_{k}$.

CASE 3. $n \equiv 2(\bmod 8), n>8:$ Let $y \in \pi_{n-1}(S O(n))$ be a class so that $i_{*}(y)$ generates $\pi_{n-1}(S O(n+1))=\mathbb{Z}_{2}$, and put $x=y-\frac{1}{2} H J(y) \partial \kappa_{n}$. Then

(3-1) $G_{n}=\mathbb{Z}_{2}$ is generated by $x$

(3-2) $J: G_{n} \rightarrow \pi_{2 n-1}\left(S^{n}\right)$ is monomorphic.

CASE 4. $n=8 s+6: G_{n}=\{0\}$.

Remark 2. In Cases 2 and $3, H J(y) \in \mathbb{Z}$ must be even for dimensional reasons.

Proof. All the statements above can be found in $\left[\mathrm{W}_{1}\right]$ except for (1-2), (2-2) and (3-2), due essentially to Adams [A] and Quillen [Q].

The $J$-homomorphisms induce the commutative diagram

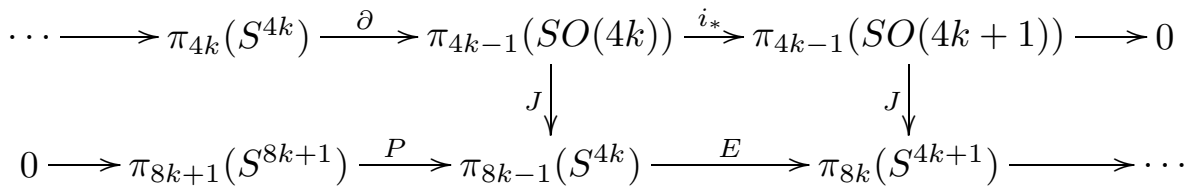

in which the bottom is a section of the EHP sequence [Wh, p. 548]. It is known that

(1) $i_{*}$ maps $G_{4 k}=\mathbb{Z}$ isomorphically onto $\pi_{4 k-1}(S O(4 k+1))=\mathbb{Z}$ if $k \geq 3$, and monomorphically onto the subgroup of index 2 if $k \leq 2$.

By Adams [A] and Quillen $[\mathrm{Q}]$ we have

(2) $J\left(\pi_{4 k-1}(S O(4 k+1))\right) \subset \pi_{8 k}\left(S^{4 k+1}\right)$ is a cyclic subgroup of order $d_{k}$ (cf. Section 1).

Combining these with the obvious fact that

(3) the composition $H \circ P: \pi_{8 k+1}\left(S^{8 k+1}\right) \rightarrow \mathbb{Z}$ is monomorphic proves (1-2) and (2-2).

Assume now that $n \equiv 2(\bmod 8)$ and $n>8$ (i.e. Case 3$)$. By Adams $[\mathrm{A}]$, $J\left(i_{*}(x)\right) \in \pi_{2 n}\left(S^{n+1}\right)$ is of order 2 . This clearly implies (3-2).

If $n$ is even and $n \neq 4 k$, then $J$ restricts to a monomorphism $G_{n} \rightarrow$ $\pi_{2 n-1}\left(S^{n}\right)$ by Lemma 4 . The homotopy classification of elements in $S(n)$ 
now coincides with the homeomorphism classification, hence was done by Wall $\left[\mathrm{W}_{1}\right]$ :

COROLlary 2. If $n$ is even and $n \neq 4 k$, we have

(1) for $n \equiv 2(\bmod 8)$ and $n>8: S(n)=\{M(0,0), M(x, 0)\}$;

(2) for $n=8 s+6: S(n)=\{M(0,0)\}$, where $M(0,0)=S^{n} \times S^{n}$.

If $n=4 k$ (Cases 1 and 2), we write $M\left(a_{1}, a_{2}\right)$ instead of $M\left(a_{1} x, a_{2} x\right)$, $a_{i} \in \mathbb{Z}$. In view of the construction of $M\left(a_{1}, a_{2}\right)$ described in Section 2, the characteristic map for the normal bundle of the embedding $\iota_{i}: S^{n} \rightarrow$ $M\left(a_{1}, a_{2}\right)$ is seen to be $a_{i} x \in \pi_{4 k-1}(S O(4 k)), i=1,2$. Thus, by the divisibility result of $\mathrm{R}$. Bott [B], the Pontryagin class $p_{k}$ for the stable tangent bundle of $M\left(a_{1}, a_{2}\right)$ is related to $\left(a_{1}, a_{2}\right)$ by the formula of Section 1 .

Proof of Theorem 2. Since $n=4 k$, the J-homomorphism restricts to the modulo- $\sigma_{k}$ reduction $G_{n}=\mathbb{Z} \rightarrow \pi_{2 n-1}\left(S^{n}\right)$ by Lemma 4 . Let $f$ : $M\left(a_{1}, a_{2}\right) \rightarrow M\left(b_{1}, b_{2}\right)$ be a homotopy equivalence. Then $r(f) \in M(2)$ must be unimodular. The congruence relations follow from (i) and (ii) of Corollary 1.

5. Proof of Theorem 3. For a self-map $f$ of a manifold $M$, let $L(f)$ be the Lefschetz number of $f[\mathrm{Br}]$. We put

$$
L(M)=\{L(f) \mid f: M \rightarrow M\} .
$$

By the definition of Lefschetz number we deduce from Corollary 1 the following

Lemma 5. If $M=M\left(a_{1}, a_{2}\right) \in T(k)$, then $L(M)=L_{1} \cup L_{2} \cup L_{3} \cup L_{4}$ with

$$
\begin{aligned}
& L_{1}=\left\{(a+1)(b+1) \mid(a b-a) a_{1} \equiv(a b-b) a_{2} \equiv 0\left(\bmod \sigma_{k}\right), a, b \in \mathbb{Z}\right\}, \\
& L_{2}=\left\{1+a b \mid a b a_{1}-b a_{2} \equiv a b a_{2}-a a_{1} \equiv 0\left(\bmod \sigma_{k}\right), a, b \in \mathbb{Z}\right\}, \\
& L_{3}=\left\{1+a \mid a a_{1} \equiv 0\left(\bmod \sigma_{k}\right), a \in \mathbb{Z}\right\}, \\
& L_{4}=\left\{1+b \mid b a_{2} \equiv 0\left(\bmod \sigma_{k}\right), b \in \mathbb{Z}\right\} .
\end{aligned}
$$

It is well known that if $M$ is a simply connected manifold, then $M$ has the fixed point property if and only if $0 \notin L(M)$.

Proof of Theorem 3. Assume $n=4 k$, and $M\left(a_{1}, a_{2}\right) \in T(k)$ (i.e. $0 \leq$ $\left.a_{1} \leq a_{2} \leq \sigma_{k} / 2\right)$. It is easy to see from Lemma 5 that the condition $a_{1} a_{2} \neq 0$ is equivalent to $0 \notin L_{3} \cup L_{4}$, and that $0 \in L_{2}$ implies $0 \in L_{1}$. We may assume below that $a_{1} a_{2} \neq 0$. Consequently, $o\left(a_{1}\right) \neq 0, o\left(a_{2}\right) \neq 0$ (since $\left.a_{1}, a_{2} \leq \sigma_{k} / 2\right)$. 
If $0 \in L_{1}$, then by Lemma 5 we have either

(i) $b=-1$ and $2 a a_{1} \equiv(a-1) a_{2} \equiv 0$, or

(ii) $a=-1$ and $(b-1) a_{1} \equiv 2 b a_{2} \equiv 0$.

However (i) implies $o\left(a_{1}\right) \mid 2 a$ and $o\left(a_{2}\right) \mid a-1$, and similarly (ii) implies $o\left(a_{1}\right) \mid b-1$ and $o\left(a_{2}\right) \mid 2 b$, both leading to either $\operatorname{gcd}\left\{o\left(a_{1}\right), o\left(a_{2}\right)\right\}=1, \operatorname{gcd}\left\{2 o\left(a_{1}\right), o\left(a_{2}\right)\right\}=2$, or $\operatorname{gcd}\left\{o\left(a_{1}\right), 2 o\left(a_{2}\right)\right\}=2$.

Conversely, if $\operatorname{gcd}\left\{o\left(a_{1}\right), o\left(a_{2}\right)\right\}=1$, so that there are $s, t \in \mathbb{Z}$ such that

$$
s o\left(a_{1}\right)+t o\left(a_{2}\right)=1 \text {, }
$$

then $(a, b)=\left(-1, o\left(a_{2}\right) t\right)$ satisfies (ii). Alternatively, if $\operatorname{gcd}\left\{2 o\left(a_{1}\right), o\left(a_{2}\right)\right\}=2$ (say), so that there are $s, t \in \mathbb{Z}$ such that

$$
2 o\left(a_{1}\right) s+o\left(a_{2}\right) t=2,
$$

then $o\left(a_{2}\right) t$ is divisible by 2 , and $(a, b)=\left(-1, o\left(a_{2}\right) t / 2\right)$ satisfies (ii). Thus $0 \in L_{1}$. The first assertion of Theorem 3 is verified.

The second assertion of Theorem 3 comes directly from the following observations:

(i) If $n$ is odd, then for $M \in S(n)$ one has

$$
L(\mathrm{Id})=\chi(M)=0 \in L(M),
$$

where Id $: M \rightarrow M$ is the identity and $\chi(M)$ is the Euler characteristic of $M$.

(ii) If $n=8 s+6$, then $S(n)$ consists of the single element $S^{n} \times S^{n}$ by Corollary 2(2).

(iii) If $n \equiv 2(\bmod 8)$ and $n>8$, then $S(n)=\left\{S^{n} \times S^{n}, M(x, 0)\right\}$ by Corollary 2(1). The matrix

$$
A=\left(\begin{array}{cc}
0 & 0 \\
0 & -1
\end{array}\right)
$$

is realizable by a self-map $f$ of $M(x, 0)$ by Corollary 1(ii); its Lefschetz number is seen to be zero.

6. Computational examples. We conclude this paper by describing some phenomena arising from the previous computation.

For every manifold $M$, the constant map and identity map of $M$ contribute to the set $L(M)$ respectively 1 and $\chi(M)$ (the Euler characteristic). Therefore, the subset $L^{\prime}(M)=L(M) \backslash\{1, \chi(M)\}$ can be viewed as the set of non-obvious Lefschetz numbers for self-maps of $M$.

If $M \in S(4 k)$, the set $L(M)$ (hence $L^{\prime}(M)$ ) may be computed by using Lemma 5. For instance, if $M=M(1,2) \in S(4 k)$, one can show that

$$
|\lambda| \geq \sqrt{\sigma_{k}} / 2 \quad \text { for all } \lambda \in L^{\prime}(M) .
$$


This estimate points out an interesting phenomenon: there exist twisted products of $S^{4 k}$ whose non-obvious Lefschetz numbers are arbitrarily large.

A fundamental invariant for a map $f: M \rightarrow N$ between two closed oriented manifolds of the same dimension is its Brouwer degree, denoted by $\operatorname{deg} f$. It may be evaluated in the following manner. Let $[M] \in H_{\operatorname{dim} M}(M)$ be the fundamental class specified by the orientation, and let $f_{*}: H_{*}(M) \rightarrow$ $H_{*}(N)$ be the induced homomorphism. In view of the fact that $H_{\operatorname{dim} M}(M)$ $=\mathbb{Z}$ is generated by $[M], \operatorname{deg} f$ is seen to be the unique integer satisfying $f_{*}[M]=\operatorname{deg} f \cdot[N]$ in $H_{\operatorname{dim} N}(N)=\mathbb{Z}$.

Given two closed oriented manifolds $M, N$ of the same dimension we set

$$
D(M, N)=\{\operatorname{deg} f \mid f: M \rightarrow N\} .
$$

The problem of determining the set $D(M, N)$ for given $M$ and $N$ can be viewed as one of the realization problems in topology, and has been studied by many authors for certain classes of 3-manifolds (cf. [S] for the latest references).

Lemma 3 is sufficient to find the set $D(M, N)$ for $M, N \in S(n)$. For instance, from Corollary 1 (a special case of Lemma 3) one finds that if $M=M^{8 k}\left(a_{1}, a_{2}\right), N=M^{8 k}\left(b_{1}, b_{2}\right) \in S(4 k)$, then

$$
\begin{aligned}
D(M, N)= & \left\{x y \mid x y b_{1}-x a_{1} \equiv 0, x y b_{2}-y a_{2} \equiv 0\left(\bmod \sigma_{k}\right)\right\} \\
& \cup\left\{x y \mid x y b_{1}-x a_{2} \equiv 0, x y b_{2}-y a_{1} \equiv 0\left(\bmod \sigma_{k}\right)\right\} .
\end{aligned}
$$

This indicates that the set $D(M, N)$ might possess interesting numerical features. Direct computations yield, as examples,

$$
\begin{aligned}
& D\left(M^{8 k}(1,1), M^{8 k}(0,0)\right)=\left\{\sigma_{k}^{2} t \mid t \in \mathbb{Z}\right\}, \\
& D\left(M^{48}(1,1), M^{48}(0,0)\right)=\left\{65520^{2} t \mid t \in \mathbb{Z}\right\}, \\
& D\left(M^{16}(1,2), M^{16}(0,0)\right)=\{7200 t \mid t \in \mathbb{Z}\}, \\
& D\left(M^{16}(1,3), M^{16}(0,0)\right)=\{4800 t \mid t \in \mathbb{Z}\}, \\
& D\left(M^{16}(1,4), M^{16}(0,0)\right)=\{3600 t \mid t \in \mathbb{Z}\} .
\end{aligned}
$$

Acknowledgements. The authors feel grateful to Mr. Qi Xin, who implemented a program enumerating all elements in $J(k)$. The results in Tables 1, 2 and 3 have been provided by him.

Thanks are also due to the referee for pointing out misprints in the earlier version of the work.

\section{References}

[A] J. F. Adams, On the groups $J(X), I-I V$, Topology 2 (1963), 181-195; 3 (1965), 137-191, 193-222; 5 (1966), 21-71.

[B] R. Bott and J. W. Milnor, On the parallelizability of the spheres, Bull. Amer. Math. Soc. 64 (1958), 87-89. 
[Br] R. F. Brown, The Lefschetz Fixed Point Theorem, Scott, Foresman, Glenview, IL, 1970.

[F] E. Fadell, Some examples in fixed point theory, Pacific J. Math. 33 (1970), 89-100.

[Fo] W. Forster, Preface, in: Numerical Solution of Highly Nonlinear Problems, W. Forster (ed.), North-Holland, 1980.

[H] M. Hoffman, Endomorphisms of the cohomology of complex Grassmannians, Trans. Amer. Math. Soc. 281 (1984), 745-740.

[Q] D. Quillen, The Adams conjecture, Topology 10 (1971), 67-80.

[S] T. Soma, Degree-one maps between hyperbolic 3-manifolds with the same volume limit, Trans. Amer. Math. Soc. 353 (2001), 2753-2772.

$\left[\mathrm{W}_{1}\right] \quad$ C. T. C. Wall, Classification of $(n-1)$-connected $2 n$-manifolds, Ann. of Math. 75 (1962), 163-198.

$\left[\mathrm{W}_{2}\right] \quad-$, Classification problems in differential topology. I, Topology 2 (1963), 253-261.

[Wh] G. W. Whitehead, Elements of Homotopy Theory, Springer, 1978.

Institute of Mathematics

Chinese Academy of Sciences

Beijing 100080, P.R. China

E-mail: dhb@math.ac.cn
Department of Mathematics

Peking University

Beijing 100871, P.R. China

E-mail: jiangbj@math.pku.edu.cn

Received 19 December 2001;

in revised form 20 October 2003 